\title{
Theory and practice of extramarital sex
}

\section{from Jared Diamond}

IN many animal species there is a lasting pair bond between a male and a female, both of whom devote much parental care to their joint offspring. Under what circumstances should such shared parental care coexist with extramarital sex, a situation that sociobiologists euphemistically term 'mixed reproductive strategy'? A recent study by Fitch and Shugart (Am. Nat. 124, $116 ; 1984)$ provides some answers.

On first reflection, one might think that males would always benefit and could never do themselves harm by pursuing a mixed strategy, because males make a much lower investment in each gamete than do females. Since the theoretical studies of Trivers (in Sexual Selection and the Descent of Man, ed. Campbell, B., 136, Aldine, Chicago; 1972), however, other factors have been appreciated. For example, extramarital sex may decrease rather than increase one's fitness if one's mate deserts as a result. A male pursuing a mixed strategy must have confidence of paternity, that is he must be assured that, while he is seeking another female, his mate is not seizing the chance to copulate with another mixed-strategy male. Perhaps the most fundamental theoretical problem is that mixed strategies cannot be considered from the standpoint of the male alone: males pursuing mixed strategies are doomed to failure in the absence of similarly inclined females. What could induce a female to pursue a mixed strategy?

Several field studies have reported evidence for mixed strategies in birds, but they have usually been based on unmarked individuals, so that it has been unclear whether participants in the supposed extramarital sex were paired, unpaired, or engaged in courting (which could lead to formation of a firm bond). That methodological problem has now been overcome by Fitch and Shugart, who marked individual herring gulls on Gull Island, Lake Michigan, and drew the following conclusions from lengthy observations.

Of the mated males studied, 35 per cent were seen to copulate with a female other than their mate. Of the mated females studied, none was seen to copulate with a male other than her mate, despite ample opportunity to do so. (For 14 per cent of the time during the fertile period, mated females were alone at the nest and a male was present on a neighbouring territory. The females did not approach that male, however, and rebuffed him if solicited). By contrast, unmated females usually did copulate if solicited.

Mated males spent more time in their territory during the period when their mate was fertile than when she was not fertile. When another bird entered the territory, the male almost always chased out the intruder. These two observations suggest that males seek confidence of paternity by guarding fertile mates, although guarding is not the sole answer as fertile females were left unguarded for 20 per cent of the time.

Thus, both male and female herring gulls pursue a mixed reproductive strategy, but males pursue both components simultaneously, while females do so sequentially. Males are accepted for extramarital sex by unmated females but not by mated females. Fitch and Shugart speculate that males induce their mates to remain faithful by diligently feeding them and by copulating frequently when the female is receptive. Ironically, none of the extramarital copulations witnessed by Fitch and Shugart resulted in an egg being laid. Thus the adaptive benefits of extramarital sex remain mysterious.

Jared Diamond is Professor of Physiology at University of California Medical School, Los Angeles, California 90024, USA.

\section{Cell biology}

\section{Microtubule catastrophe}

\section{from J. Richard McIntosh}

Microtubules are an important part of the system of cytoplasmic fibres that defines the shape of a cell and helps it to move around. Observations of these fibers with several kinds of microscopes have demonstrated that cells modulate the organization of their microtubules during the cell cycle. For example, the microtubules that are arrayed in the cytoplasm of the cell in interphase disassemble into tubulin subunits as the mitotic spindle forms. Two papers by $\mathbf{T}$. Mitchison and M. Kirschner in this issue ${ }^{1,2}$ cast the lability of microtubules in a new light. Under certain conditions in vitro, a population of microtubules will behave in two ways at once: some polymers elongate while others catastrophically disassemble and disappear.

It is widely believed that the positions of microtubules in cells are defined by special 'organizing centers' composed of macromolecules other than tubulin. A cell modulates the activity of these centres to define the number of microtubules that form. The centrosome is probably the most important microtubule organizer in an animal cell. This organelle was recognized years ago as a regulator of cellular morphogenesis, but modern work on centrosomes has been sparse, in part because they are difficult to isolate for detailed study. On page 232 Mitchison and Kirschner present a method for preparing active centrosomes from cultured mammalian cells and describe some of the properties of the microtubule arrays they initiate. This study confirms earlier work on centrosome activity by showing that the number of microtubules initiated depends on the concentration of tubulin used for assembly ${ }^{3-5}$. If the subunit concentration exceeds a critical value, microtubules will form, as expected from the 'condensation-polymerization' model of Oosawa and $\mathrm{Kasai}^{6}$ but, unlike most phase transitions, the number of polymers formed increases rather slowly with increasing concentrations of subunits, saturating at roughly five times the critical concentration.

Mitchison and Kirschner go on to identify properties of their system that are incompatible with the formulation by Oosawa and Kasai. The variation in microtubule number with the concentration of soluble tubulin is not simply a consequence of the amount of tubulin present at the time of polymer initiation. When microtubules initiated from a centrosome at high tubulin concentration (lots of microtubules per organizing centre) are diluted into an intermediate concentration of tubulin, the number of microtubules per centrosome decreases to that appropriate for the intermediate concentration. Even more surprising, while some microtubules are disappearing, the ones that are left get longer.

One might imagine that this behaviour reflects some unusual property of centrosome-initiated microtubules, but in their second paper, Mitchison and Kirschner show analogous phenomena for microtubules formed by spontaneous initiation in solution. Again, the distribution of lengths in a preparation of microtubules changes upon dilution, with some fibres elongating, while others disappear. At polymerization steady state, a constant polymer mass is maintained by the slow elongation of ever fewer microtubules. This view of the steady state is completely different from the one presented by Margolis and Wilson ${ }^{7}$, which holds that subunits add to one end of each polymer and fall off from the other, a process often called treadmilling. It is too soon to evaluate these two views of the steady state, but the distinctions between their predictions are very clear. Discrimination by experiment should soon be possible.

To interpret their data, Mitchison and Kirschner use a model based on studies of GTP binding to tubulin ${ }^{8-11}$. GTP that 